\title{
Experimental electron Compton profile beyond the impulse approximation. Comparison of results on $\mathrm{NH}_{3}$ and $\mathrm{Ne}\left({ }^{*}\right)$
}

\author{
A. Duguet, A. Lahmam-Bennani and M. Rouault \\ Laboratoire des Collisions Atomiques et Moléculaires ( $\left.{ }^{* *}\right)$, Bât. 210, Université Paris-Sud, F 91405 Orsay Cedex, France
}

(Reçu le 15 janvier 1980, révisé le 6 mai, accepté le 30 mai 1980)

\begin{abstract}
Résumé. - La partie symétrique du profil Compton du néon, déterminée par diffusion d'électrons de $25 \mathrm{keV}$, est comparée aux calculs théoriques suivant l'approximation de l'impulsion, et la partie antisymétrique est comparée aux prévisions qualitatives de Tavard pour des orbitales hydrogénoïdes. Les résultats antérieurs sur $\mathrm{NH}_{3}$ sont rappelés et une tentative d'interprétation physique est donnée.
\end{abstract}

Abstract. - The symmetrical part of the neon Compton profile, obtained by $25 \mathrm{keV}$ electron scattering is compared to impulse approximation calculations, and the antisymmetrical part is compared to a qualitative estimate by Tavard for hydrogenic orbitals. Previous results on $\mathrm{NH}_{3}$ are recalled and a physical interpretation is attempted.

Within the framework of the impulse approximation (IA), the Compton profile (CP) of any target peaks at the energy-loss, $E_{\mathrm{IA}}$, corresponding to the scattering by a free and stationary electron. The existence of a Compton shift, $\Delta E=E_{\exp }-E_{\mathrm{IA}}$, where $E_{\exp }$ is the observed energy-loss at the maximum of the CP, was established by Barlas et al. [1]. However, we have shown in recent experiments $[2,3]$ that this Compton shift is not sufficient to describe the deviations of the $\mathrm{NH}_{3} \mathrm{CP}$ from the IA predictions. The purpose of this note is to report similar deviations as recently measured for neon and to compare them to the $\mathrm{NH}_{3}$ results which will be briefly recalled.

The $\mathrm{CP}$ of $\mathrm{NH}_{3}, J(q, K)$, was determined at different scattering angles $\left({ }^{1}\right)$, and it was found to depend not only on the energy-loss $E$, through

$$
\dot{q}=\left(2 E-K^{2}\right) / 2 K\left({ }^{2}\right),
$$

(*) La traduction en français de cet article a été acceptée aux Comptes Rendus de l'Académie des Sciences et elle est insérée dans le $\mathrm{n}^{\circ} \mathrm{du} 4$ février 1980.

$\left.{ }^{* *}\right)$ Associé au C.N.R.S. $\mathrm{n}^{\circ} 281$.

( $\left.{ }^{1}\right)$ Bonham has shown [8] that, along a profile at constant scattering angle, the momentum transfer $K$ is very close to its value at the maximum of the profile.

${ }^{2}$ ) All quantities are here expressed in atomic units. The expression for $q$ in Ref. [2] is correct if the energy is evaluated in Rydberg (1 a.u. $=2$ Ry). but also on the momentum transfer $K$, while the corresponding quantity $J_{\mathrm{IA}}(q)$, as predicted according to the IA, is only a function of $q$. However there are two $K$-ranges where the experimental profile is practically independent of $K$; for $K>6$ a.u., one gets a total profile $J^{\mathrm{T}}(q)$ due to electrons ejected from all orbitals of the molecule; for 3 a.u. $<K<5$ a.u., it is a valence profile $J^{\mathrm{v}}(q)$ due to electrons ejected in collisions where the $1 \mathrm{a}_{1}$ electrons of $\mathrm{NH}_{3}$ are excluded. In each of these ranges, the symmetrical part $J_{s}(q)=(J(q)+J(-q)) / 2$ is in good agreement with the theoretical corresponding IA profile, respectively $J_{\mathrm{IA}}^{\mathrm{T}}(q)$ and $J_{\mathrm{IA}}^{\mathrm{v}}(q)$; the antisymmetrical part $J_{\mathrm{a}}(q)=(J(q)-J(-q)) / 2$ is then a good representation of the second order term of the profile, that is total profile for $K>6$ a.u. and valence profile for 3 a.u. $<K<5$ a.u. ; by subtraction, one can obtain the symmetrical and the antisymmetrical parts, $J_{\mathrm{s}}^{1 \mathbf{a}_{1}}(q)$ and $J_{\mathrm{a}}^{1 \mathrm{a}_{1}}(q)$, corresponding to ionizing collisions on the $\mathrm{NH}_{3} 1 \mathrm{a}_{1}$ orbitals formed by the nitrogen $1 \mathrm{~s}$ orbitals.

Electron CP's have been obtained for neon from energy-loss spectra of $25 \mathrm{keV}$ electrons scattered in the angular range corresponding to momentum transfer values from 1 to 15 atomic units [4]. Figure 1 shows, for two scattering angles, the cross section doubly differential in angle and energy-loss, $\mathrm{d}^{2} \sigma / \mathrm{d} E \mathrm{~d} \Omega$. For $K<7$ a.u. (Fig. $1 a$ ), the main profile, up to $E \approx 870 \mathrm{eV}$, is due to the scattering 


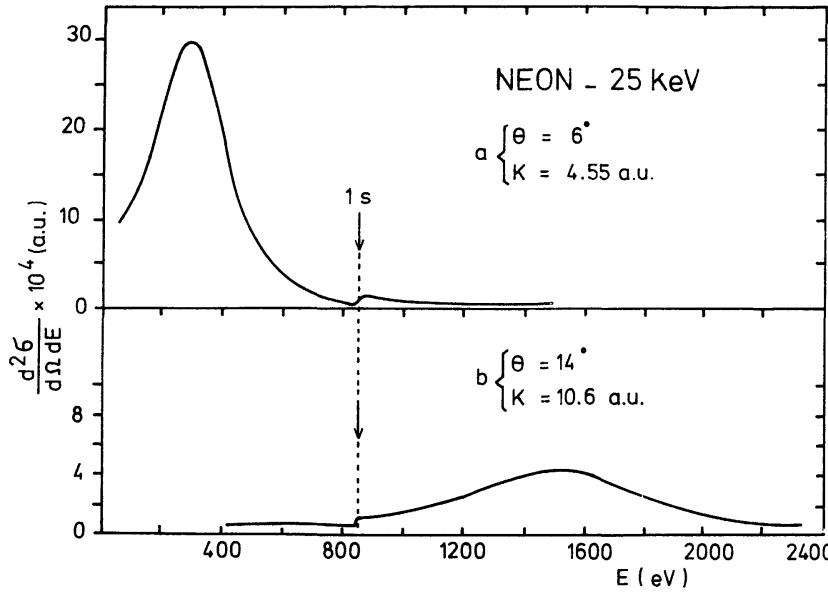

Fig. 1. - Cross sections, $\mathrm{d}^{2} \sigma / \mathrm{d} E \mathrm{~d} \Omega$, differential with respect to the angle and the energy-loss for inelastic scattering of electrons from : $a$ ) the L-electrons, and $b$ ) all the electrons of neon.

by the L-electrons ( $2 s$ and $2 p)$ of the neon atom; it is a valence profile. For $K>10$ a.u. (Fig. $1 b$ ), all neon electrons (1s, 2s, 2p) are involved in the scattering process; it is a total profile. A profile is usually represented by $J(q, K)=\frac{K^{5}}{2} \frac{k_{0}}{k} \frac{\mathrm{d}^{2} \sigma}{\mathrm{d} E \mathrm{~d} \Omega}\left(k_{0}\right.$ and $k$ are the initial and final momenta of the colliding electron). Figure 2 represents the maximum value of each profile, $J_{\max }(q, K)$, as a function of $K$. In the framework of the IA, this maximum is simply the expectation value $N\left\langle p^{-1}\right\rangle$ relative to the momenta $p$ of the $N$ target electrons contributing to the profile. There are two plateaus in the $K$-ranges 4 a.u. $<K<8$ a.u. and 8 a.u. $<K$, where the IA is a good first order approximation, respectively for the valence profile $(N=8$, first range) and the total profile $(N=10$, second range) [3].

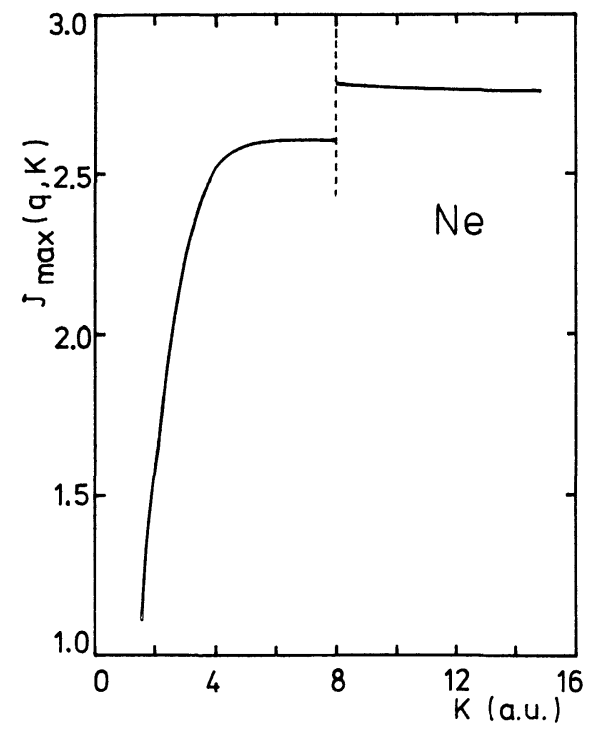

Fig. 2. - The maximum value, $J_{\max }(q, K)$, of the Ne electron CP versus momentum transfer $K$.
Using the mean profile, $J(q)$, as determined in each of these two regions for $K$ values not too close to the limits, we have calculated $J_{\mathrm{s}}(q)$ and $J_{\mathrm{a}}(q) . J_{\mathrm{s}}(q)$ is in good agreement with $J_{\mathrm{IA}}(q)$ calculated in the framework of the IA [5], with deviations smaller than respectively $2 \%$ for the valence profile up to $q=1.5$ a.u. and $1.9 \%$ for the total profile up to $q=2.0$ a.u.. The antisymmetrical part $J_{\mathrm{a}}(q)$ is represented in figure 3 for the total profile, the valence profile, and their difference which gives the K-shell profile (1s orbitals). These curves are in accordance with the theoretical qualitative predictions of Tavard for hydrogenic orbitals [6].

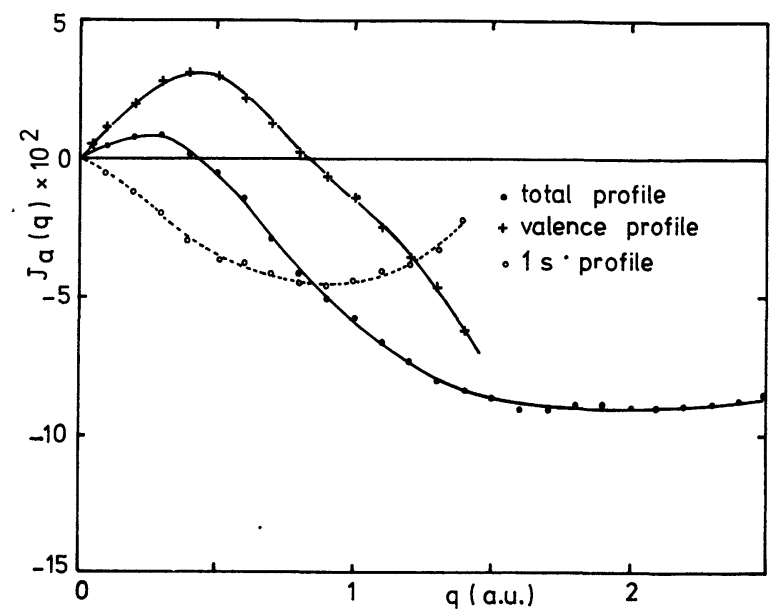

Fig. 3. - Antisymmetrical parts of the total, valence, and 1s $\mathrm{Ne}$ electron CP's, respectively $J_{\mathrm{a}}^{\mathrm{T}}(q), J_{\mathrm{a}}^{\mathrm{V}}(q)$ and $J_{\mathrm{a}}^{1 \mathrm{~s}}(q)$. The incident electron energy is $25 \mathrm{keV}$.

The $\mathrm{NH}_{3}$ antisymmetrical experimental profiles [3] (Fig. 4) behave differently, especially the nitrogen $\mathrm{K}$-shell profile, whose sign is opposite to that of neon,

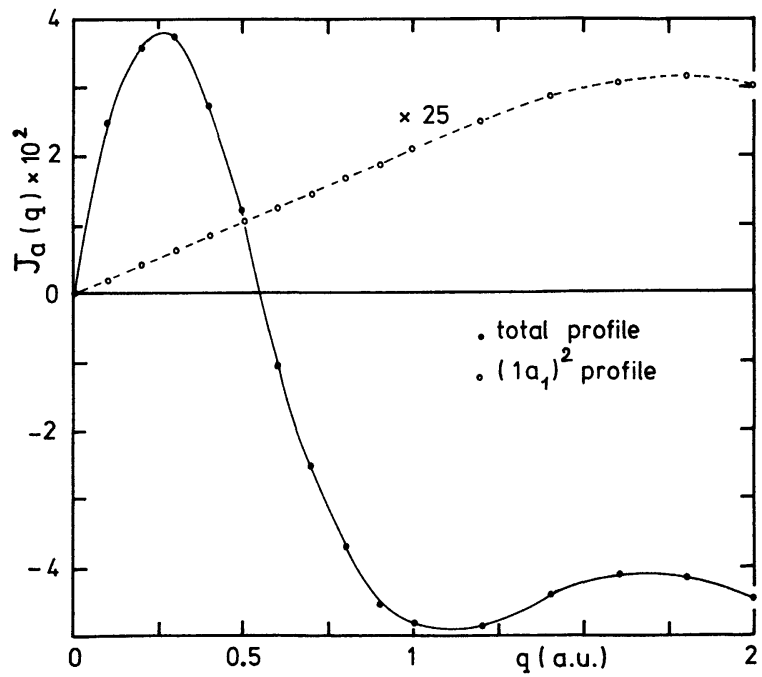

Fig. 4. - Same as in figure 3 but for $\mathrm{NH}_{3}$, with an incident electron energy of $35 \mathrm{keV}$. 
and whose magnitude is about fifty times lower at the maximum; the antisymmetrical total and valence profiles are then very close to each other.

According to Tavard's interpretation, the electron $\mathrm{CP}, J(q, K)$, can be written [7] as the sum of a first term which exactly represents the IACP, $J_{\mathrm{IA}}(q)$, and of a second order term related to some integrals of the potential interaction type (see Ref. [7] for the exact equations). Qualitative predictions can be obtained by assuming that the energy-losses are large compared to the ionization energies involved, and mainly by taking into account the action of localized charges on the ejected electrons, i.e. by neglecting in a first step the electron-electron interactions compared to the electron-nucleus interactions. The electron-electron interactions are of opposite sign compared to the electron-nucleus ones and they are not localized, hence they are equivalent to a lowering of the nucleus effective charge. For neon, this lowering does not exceed the number of non-ejected electrons, $Z-1$, the effective charge is then positive and larger than 1 . For $\mathrm{NH}_{3}$, the lowering of the effective charge is due to the $Z-1$ electrons of $\mathrm{N}$ and to the transfer of electronic charge from the three hydrogen to the central atom occurring during the chemical bond formation; the six bond electrons ( $3 \mathrm{p}$ electrons from $\mathrm{N}$ and 3 electrons from $\mathrm{H}$ ) being quite localized may then sufficiently lower or even make slightly negative the nitrogen effective charge. This can explain the difference in sign and order of magnitude between the $\mathrm{NH}_{3}$ and the $\mathrm{Ne}$ antisymmetrical profiles. It is also to be noted that a $\mathrm{K}$-electron of $\mathrm{N}$ contributing to the $\mathrm{la}_{1}-\mathrm{NH}_{3}$ profile is at a large distance from the $\mathrm{H}$ nuclei $\left(r_{\mathrm{NH}} \approx 1 \AA\right.$; radius of the $\mathrm{K}$-orbit $r_{1 \mathrm{sN}} \approx 0.1 \AA$ ), and hence the action of these nuclei are negligible.

It is to be noted that in this research, profiles could be obtained only in experimental conditions where the energy-loss at the maximum of the profile is not very large compared to the ionization energy of the concerned shell (10 times for the valence shell, but only 3 times for the $\mathrm{K}$-shell); this is not very favourable for theoretical interpretation.

Experimental work on $\mathrm{H}_{2} \mathrm{O}$ and $\mathrm{CH}_{4}$ as well as further theoretical studies seem to be desirable in this domain.

Acknowledgments. - Fruitful discussions with C. Tavard throughout this work are acknowledged.

\section{References}

[1] Barlas, A. D., Rueckner, W. H. E., Wellenstein, H. F., J. Phys. B 11 (1978) 381-400.

[2] I umam-Bennani, A., Duguet, A., Rouault, M., Philos. Mag. B 38 (1978) 95.

[3] Lahmam-Bennani, A., Duguet, A., Wellenstein, H. F., Rouault, M., J. Chem. Phys., accepted for publication (1980).

[4] Duguet, A., Lahmam-Bennani, A., Rouault, M., to be published (1980).
[5] Tong, B. Y., Lam, L., Phys. Rev. A 18 (1978) 553.

[6] TAVARD, C., French-german meeting on electron Compton scattering, Université de Metz, oct. 1978.

[7] Gasser, F., Tavard, C., C. R. Hebd. Séan. Acad. Sci. Paris 286B (1978) 13.

[8] Bonham, R. A., J. Chem. Phys. Lett. 18 (1973) 454. 